\title{
On the Prediction of Structural Layout for Maximum Stiffness ${ }^{1}$
}

\author{
J. E. TAYLOR ${ }^{2}$
}

Dedicated to Professor W. Prager

\begin{abstract}
A variational formulation is presented for the problem of predicting the optimal structural system from among a discrete set of possible designs. The development is written for maximum stiffness design of single-purpose structures. The distribution among admissible structural configurations of the prescribed loads is determined as part of the solution. An extremum problem interpretation also provides the basis for evaluating relative efficiencies among locally optimal structural systems.
\end{abstract}

Key Words. Calculus of variations, structural optimization.

\section{Introduction}

Direct methods for the numerical evaluation of solutions prove to be inadequate for large-scale structures optimization problems. For certain problems, the computational effort associated with such methods simply becomes prohibitive. Prager (Ref. 1) discusses several other sources of difficulty. For example, the use of first-order methods may result in convergence to a nonoptimal point. Also, in layout design problems, care must be taken to avoid the inadvertent exclusion of the optimal solution through an inappropriate statement of the problem (Ref. 2).

A lowered dimension of the design space (basis) might be established as one means to reduce the demands on means for computation. Sheu and Schmit (Ref. 3) demonstrate a result in this direction. Nagtegaal and

${ }_{1}$ The work reported in this paper was supported by the National Science Foundation, Grant No. GK-31476.

${ }^{2}$ Associate Professor, Department of Aerospace Engineering, and Department of Applied Mechanics and Engineering Science, University of Michigan, Ann Arbor, Michigan. 
Prager (Ref. 4) establish a limited superposition principle, also toward lowering the scale of the design problem.

This paper presents an extended variational formulation for the problem of optimal layout and member sizing, for maximum stiffness under a single configuration of load. The approach is reminiscent of techniques used in recent years by Prager, Drucker and Shield, Shield and Prager, Masur, Mroz, Martin, and Taylor (Refs. 6-11) among others, where the variational form relates closely to classical extremum principles of analysis. Additional unknowns, the unit relative cost indices, are introduced to facilitate the treatment of the system-design problem effectively through a single functional. It is proved that the solution of the problem is identified with the single (unique) stationary point of this functional.

\section{Candidate Structural Systems}

The name structural system designates any design that is capable of sustaining the entire set of prescribed loads. The following development exemplifies the design problem of predicting the most efficient one among an arbitrary finite number of feasible systems. In the case of trussed structures, the problem statement may be comprised of: (a) a listing of the prescribed forces (loads); (b) a definition of the design space, in the form of a designation of available internal and support nodes; and (c) a set of unit cost factors, taken here as the cost per unit volume of material; cost factors are specified independently for each system.

Any combination of members able to carry one or more components of load is labeled a configuration. All systems consistent with the design space (b) are to be considered, i.e., every possible combination of statically determinate and indeterminate configurations belongs to the set of feasible systems. Note, also, that the external forces at a given point may be proportioned among separate configurations which intersect at that node. The specific design problem to be treated here takes the form: predict the stiffest structural system to carry a given set of forces at a specified total cost. A solution to this problem includes the identification of layout and load distribution for the optimal system, the prediction of member sizes in the system, and the determination of structural response (deformations).

The problem is formulated in a way to accommodate an arbitrary distribution of the prescribed loads. In other words, the proportioning of external forces $P_{\beta}$ into components $f_{\beta s}^{(k)}$ per $k$ th 
configuration is arbitrary within the constraint of statical equivalence

$$
\sum_{k=1}^{N_{s}} f_{\beta s}^{(k)}=P_{\beta}, \quad \beta=1,2, \ldots, M .
$$

The number $N_{s}$ of configurations may be anywhere from 1 to the maximum number possible within the prescribed design space. The range $M$ of $\beta$ equals (for space trusses) three times the number of loaded nodes. Conditions of equilibrium for separate configurations will be expressed in the usual way through stationarity relative to response of the respective potential energies. If $\eta_{i}^{(h)}$ represents the specific strain energy of the $i$ th member out of $J_{k}$ members in configuration $k$, the configuration strain energy is

$$
U_{k s}=\sum_{i=1}^{J_{k}} l_{i} B_{i s}^{(k)} \eta(k)
$$

with $B_{i s}^{(k)}$ and $l_{i}$ as the member area and length. Subscript $s$ is to identify structural system. Structural response is measured by the generalized displacements $u_{\beta s}^{(k)}$. Thus, the loss in potential of forces $f_{\beta s}^{(k)}$ is given by

$$
W_{k s}=\sum_{\beta=1}^{M} f_{B s}^{(k)} u_{\beta s}^{(h)}
$$

and the configuration potential energy $E_{k s}$ is simply $E_{k s}=U_{k s}-W_{k s}$. The sth one among possible systems is modelled through functional $F_{s}$, formed by summing $E_{k s}$ over all configurations in the system, and augmented by constraints (1), that is,

$$
F_{s}=\sum_{k=1}^{N_{s}} E_{k s}-\sum_{\beta=1}^{M} \mu_{\beta s}\left(P_{\beta}-\sum_{k=1}^{N_{s}} f_{B s}^{\{k}\right)
$$

Functional (4) serves the function of potential energy in the conventional equilibrium problem statement. That is, equilibrium of system $s$ is identified in the usual way with stationarity of $F_{s}$ relative to response $u_{\beta s}^{(k)}$. Note, however, that the distribution factors $f_{\beta s}^{(k)}$ of prescribed forces $P_{\beta}$ appear as unknowns in the functional.

The dependence of efficiency on member size is expressed through cost. The total cost $C_{s}$ of system $s$ in terms of unit cost $r_{s}$ is given by

$$
C_{s}=r_{s} \sum_{k=1}^{N_{s}} \sum_{i=1}^{J_{k}} B_{i s}^{(k)} l_{i}
$$


Systems of equal cost, say $C$, are to be compared so that all systems are constrained by

$$
r_{s} \sum_{k=1}^{N_{s}} \sum_{i=1}^{J_{k}} B_{i s}^{(i k)} l_{i}=C, \quad s=1,2, \ldots
$$

Therefore, the system design problem is based on the functional

$$
G_{s}=F_{s}+A_{s}\left(C-r_{s} \sum_{k=1}^{N_{s}} \sum_{i=1}^{J_{k}} B_{i s}^{(k)} l_{i}\right)
$$

$G_{s}$ is a function of $B_{i s}^{(k)}$, as well as the prior $f_{\beta s}^{(k)}$ and $u_{\beta s}^{(k)}$. Stationarity of $G_{s}$ requires, for each one among all candidates for the optimal structural system, that the following equilibrium and optimality equations be satisfied:

$$
\begin{aligned}
\sum_{i=1}^{J_{i s}} B_{i s}^{(k)} l_{i} \partial \eta_{i s}^{(k)} / \partial u_{\beta s}^{(i s)}-f_{\beta s}^{(k)} & =0, \\
u_{\beta s}^{(k)}-\mu_{\beta s} & =0, \\
\eta_{i s}^{(k)}-r_{s} A_{s} & =0,
\end{aligned}
$$

as well as constraint equations (1) and (5). Equations (7) and (8) hold for all $\beta$ and $k$, while (9) applies for all $i$ and $k$.

Note that the optimality equations (8) and (9) are expressed in terms of kinematic variables alone. This uncoupling in the system of equations makes it possible to identify a priori the form of their solution. Toward this end, recognize from Eq. (9) that the structure making up each configuration is statically determinate. This follows from the fact that the equation can be satisfied in general for no greater number of members than there are degrees of freedom in the configuration. The result is combined with Eq. (8) to conclude that at most one configuration is in contact with each interior node. This is because Eq. (8) limits the solution for response $u_{\beta s}^{(k)}$ to only one set of independent displacements.

As a consequence, each component $P_{\beta}$ of external force is carried solely by one configuration. If, for example, the $\gamma$ th component is carried by the $l$ th configuration, then

$$
f_{\gamma}^{(k)}= \begin{cases}P_{\gamma} & \text { for } \quad k=l \\ 0 & \text { for } \quad k \neq l\end{cases}
$$

To summarize, if a solution (or solutions) to the system of equations for stationarity of $G_{s}$ exists, it is generally associated with a structural 
system comprised of a set of one or more statically determinate configurations, where the set covers all loaded nodes without overlap. One is enabled by this result to establish easily enough the count of such candidate structural systems as well as their form. Thus, both the nature and number of them are predicted from the analysis so far. In effect, all that remains to complete the solution is to compare the efficiencies of these systems.

\section{Level Unit Cost Formulation}

The original design task corresponds to a nonlinear programming problem in $\Phi$-dimensional design space, where $\Phi$ equals the sum of the number of members possible in all feasible systems. Each candidate system corresponds to a local extremum in this space. The problem is reduced via the argument of the prior section to a set of separate problems; the dimension of each corresponds to the extent of the respective system.

It is demonstrated in what follows that the comparison of efficiency among the systems (local extrema) may be achieved by solving another variational problem. As a first step in the development of this alternative formulation, an argument is presented to verify that candidate systems may be compared on the basis of certain unit relative cost indices. Next, the evaluation of these unit cost indices is identified with a simple extremum problem (expressed in terms of strain energies). Ultimately, this and the prior formulations are combined into a single variational statement of the original design problem.

The measure of comparison between any two candidate structural systems is taken to be the unit relative cost index, defined in terms of the unit cost factors $r$. This factor, say $\Pi_{Q P}$ for the comparison of the $P$ th and $Q$ th systems, is

$$
\Pi_{O P}^{2}=r_{Q} / r_{P} .
$$

The value of $\Pi_{Q P}$ for given unit costs is to be compared with the value, say $\tilde{\Pi}_{Q P}$, of the unit relative cost factor such that the two systems $P$ and $Q$ are of equal compliance for equal total cost. Such values are identified as the level unit relative cost indices (LURCI). Then, cost $P \gtrless \operatorname{cost} Q$, according to whether $\Pi_{Q P} \lessgtr \tilde{\Pi}_{Q P}$.

To see this, note that by the condition of equal cost, the volume of material in the systems are related by

$$
\tilde{V}_{P} \mid \tilde{V}_{Q}=\tilde{\Pi}_{O P}^{2}
$$


If other $P$ and $Q$ systems of equal stiffness are compared to the level cost ones,

$$
\tilde{V}_{P} / \tilde{V}_{Q}=V_{P} / V_{Q}
$$

so that

$$
C_{Q} / C_{P}=r_{Q} V_{Q} / r_{P} V_{P}=\left(\Pi_{Q P} / \tilde{\Pi}_{Q P}\right)^{2},
$$

and the statement is verified. To facilitate making all the comparisons generally required, note that, given the solution $\widetilde{\Pi}_{O P}, \widetilde{\Pi}_{R P}, \ldots$, for all candidate systems compared to the $P$ th one, other LURCI values are obtained from

$$
\tilde{\Pi}_{R Q}=\tilde{\Pi}_{R P} / \tilde{\Pi}_{Q P}, \ldots
$$

Thus, comparisons among the candidate systems may be made sequentially, solely on the basis of the set of LURCI values.

The values themselves may be calculated directly if the optimal material distribution within each candidate system is already known. Suppose that such distributions, say $\bar{A}_{i s}$ for system $s$, are determined within the normalization

$$
\sum_{i} \bar{A}_{i s} l_{i}=C / r
$$

in other words, for equal total cost $C$ and equal unit cost $r$. Any other locally optimal system is then represented by

$$
A_{i s}=\gamma_{s} \bar{A}_{i s} \text {. }
$$

The specific energy, say $\bar{\eta}_{s}$, for optimal system $\bar{A}_{i s}$ under given load is taken to be known as well. Comparing proportional systems $A_{i s}$ and $\bar{A}_{i s}$ under the same load,

$$
\eta_{s}=\bar{\eta}_{s} / \gamma_{s}^{2}
$$

so that

$$
U_{s}=\eta_{s} \sum_{i} A_{i s} l_{i}=\left(\bar{\eta}_{s} / \gamma_{s}^{2}\right) \sum_{i} \gamma_{s} \bar{A}_{i s} l_{i}=\left(\tilde{\eta}_{s} / \gamma_{s}\right) C / r
$$

Thus, to relate equal-compliance systems $P$ and $Q$,

$$
U_{P}=\left(\bar{\eta}_{P} / \gamma_{P}\right)(C / r)=\left(\bar{\eta}_{Q} / \gamma_{Q}\right) C / r=U_{Q},
$$

or

$$
\gamma_{P} / \gamma_{Q}=\bar{\eta}_{P} / \bar{\eta}_{Q}
$$


Also, the cost of a given system is

$$
C_{s}=r_{s} \sum_{i} A_{i s} l_{i}=r_{s} \gamma_{s} \sum_{i} \bar{A}_{i s} l_{i}=r_{s} \gamma_{s} C / r,
$$

so that, for equal cost $C_{P}=C_{Q}$,

$$
r_{p} \gamma_{p}=r_{Q} \gamma_{Q}
$$

or

$$
\gamma_{P} / \gamma_{Q}=\gamma_{Q} / r_{P}=\Pi_{O P}^{2}
$$

Eliminating $\gamma$ between Eqs. (17) and (18), we have

$$
\tilde{\Pi}_{Q P}^{2}=\bar{\eta}_{P} / \bar{\eta}_{Q}=\tilde{\eta}_{O} \tilde{\eta}_{P} .
$$

The latter relation follows from the first and Eqs. (16) and (18); the tilde identifies the equal-cost, equal-energy solution. To summarize, Eq. (19) states that, for equal-cost, equal-energy systems, (i) the LURCI equals the (ratio) ${ }^{-1}$ of the specific energies of the normalized systems, and (ii) the actual system specific energies are proportional to the LURCI value.

The procedure just outlined may be identified with a simple minimization problem, which in turn suggests a possible direct method for computation of the LURCI factors. This problem is stated as follows.

Determine the proportioning of material between two optimal systems $P$ and $Q$ under identical loads and with specified total cost, such that the total strain energy of the two systems is a minimum.

The total cost requirement

$$
C_{P+O}=r_{P} \sum_{i} A_{i P} l_{i}+r_{Q} \sum A_{i Q} l_{i}
$$

is transformed using Eq. (15) to

where

$$
\gamma_{P}+\Pi_{Q P}^{2} \gamma_{Q}=\bar{C}
$$

$$
\bar{C}=\left(C_{P_{+} O} / r_{P}\right) /(C / r)
$$

represents nondimensional cost.

Minimization of total strain energy within constraint [Eq. (20] is identified with the functional

$$
J=U_{P}+U_{Q}-\Gamma\left[\bar{C}-\left(\gamma_{P}+\Pi_{Q P}^{2} \gamma_{Q}\right)\right] .
$$

The individual system strain energies $U_{p}$ and $U_{o}$ are given by Eq. (16). 
Note that normalized specific strain energy $\bar{\eta}$ is independent of $\gamma$. The necessary conditions for a minimum of $J$ relative to $\gamma_{P}$ and $\gamma_{Q}$ are

$$
\left(\bar{\eta}_{P} / \gamma_{P}^{2}\right) C / r-\Gamma=0, \quad\left(\bar{\eta}_{O} / \gamma_{O}^{2}\right) C / r-\Pi_{O P}^{2} \Gamma=0 .
$$

The solution of Eqs. (22) and (20) provides

$\left(\hat{\gamma}_{P} / \hat{\gamma}_{Q}\right)=\Pi_{Q P} \sigma_{P Q}, \quad \hat{\gamma}_{P}=\bar{C}_{\sigma_{P Q}} /\left(\sigma_{P Q}+\Pi_{Q P}\right), \quad \hat{\gamma}_{Q}=\bar{C}\left[\left[\Pi_{Q P}\left(\sigma_{P Q}+\Pi_{Q P}\right)\right]\right.$,

where $\sigma_{P Q}^{2}=\bar{\eta}_{P} / \bar{\eta}_{Q}$ is introduced for convenience. If arbitrary material distribution $\gamma_{P}, \gamma_{Q}$ is related to this solution through differences $p$ and $q$, that is,

$$
\gamma_{P}=\hat{\gamma}_{P}+p, \quad \gamma_{Q}=\hat{\gamma}_{Q}+q,
$$

then an expansion of

$$
U_{P+Q}=U_{P}+U_{Q},
$$

taking account of Eqs. (30) and (23), leads to

$$
U_{P+Q}-\hat{U}_{P+Q} \sim\left(\sigma_{P Q}^{2}+\Pi_{P Q} \sigma_{P Q}\right) \Pi_{P Q}^{2} q^{2} \geqslant 0 .
$$

Thus, Eqs. (22) are sufficient as well as necessary for a minimum of the total strain energy of the two systems.

If the first of Eqs. (23) is combined with the equal-energy condition, [Eq. (17)], one obtains

$$
\tilde{\Pi}_{Q P}=\sigma_{P Q}
$$

Comparison of this result with Eq. (19) of the prior development verifies the equivalence of the two problem statements. That is, equalcost + equal-stiffness as a basis for the determination of (i) the proportion between equally loaded locally optimal systems of a total amount of material and (ii) the associated LURCI values is equivalent to minimization of total strain within given cost + the equal-energy condition.

The parallel problem is expressed in terms of complementary strain energy as follows.

Determine the proportioning of material between two internally optimal systems $P$ and $Q$ under identical loads and with specified total cost, such that the total complementary strain energy is a minimum.

It may be verified in the same way. The complementary strain energy $U_{s}{ }^{*}$ for system $s$ is written in the sameform as the prior strain energy:

$$
U_{s}^{*}=\left(\bar{\eta}_{s}^{*} / \gamma_{s}\right) C / r
$$


The governing functional $J^{*}$ matches $J$ of $\mathrm{Eq}$. (21) with $U$ replaced by $U^{*}$, and the steps leading to the counterpart of Eqs. (23) and the proof of sufficiency follow the earlier development. Of course, the result

$$
\tilde{\Pi}_{Q P}=\sigma_{P Q}^{*}=\sqrt{\left(\bar{\eta}_{P} * / \bar{\eta}_{Q}^{*}\right)}
$$

corresponds to Eqs. (19), (24), since the values of $\sigma$ and $\sigma^{*}$ are equal at the solution point.

The comparison problem for a set of $n$ candidate systems may also be set up in the same way. The functional $J$ taking the place of Eq. (21) is

$$
J=\sum_{R=1}^{n} U_{R}-\Gamma\left(\tilde{C}-\sum_{R=1}^{n} \Pi_{R 1} \gamma_{R}\right)
$$

and the associated Euler-Lagrange equations are

$$
\left(\bar{\eta}_{R} / \hat{\gamma}_{R}^{2}\right) \bar{C} / r-\Gamma \Pi_{R I}^{2}=0, \quad R=1,2, \ldots, n .
$$

From Eqs. (28), the definition of $\sigma$, and noting that $\Pi_{11}=1$,

$$
\hat{\gamma}_{R}=\hat{\gamma}_{1} / \Pi_{R 1} \sigma_{1 R}
$$

Equations (29) and the total cost constraint lead to

$$
\hat{\gamma}_{R}=\bar{C} /\left[\Pi_{R 1} \sigma_{1 R} \sum_{R=1}^{n}\left(\Pi_{R 1} / \sigma_{1 R}\right)\right], \quad R=1,2, \ldots, n .
$$

This result is substituted into the equal-energy condition

to obtain

$$
\bar{\eta}_{s / \gamma_{s}}=\bar{\eta}_{T} / \gamma_{T}
$$

$$
\tilde{\Pi}_{T s}=\sigma_{s T}, \quad s, T=1,2, \ldots, n,
$$

which compares to Eq. (24).

Combined Formulation. Suppose that, for a given design space, the candidate systems are identified according to the results of Section 2. Then, both the determination of the optimal material distribution and the evaluation of the LURCI parameters are associated with the stationarity of the functional

$$
\begin{aligned}
I\left(\bar{A}_{i s}, u_{\beta s}, \gamma_{s}\right)= & \sum_{s}\left\{\left(1 / \gamma_{s}\right) \sum_{i} l_{i s} \bar{A}_{i s} \bar{\eta}_{i s}-\sum_{\beta} P_{\beta} u_{\beta s}-A\left(\sum_{i} \bar{A}_{i s} l_{i s}-1\right)\right\} \\
& -\Gamma\left(\bar{C}-\sum_{s} \Pi_{s 1}^{2} \gamma_{s}\right) .
\end{aligned}
$$


As before, indices $s, \beta, i$ identify system, generalized displacement, and member, respectively. A single value of $A$ for all systems assures that the equal-energy requirement is met.

As an example, the trusses and cantilever beam included in the design space designated in Fig. 1(a) are evaluated. Three candidate systems are involved [Figs. 1(b), 1(c), 1(d)]. The beam is modelled as an idealized sandwich beam with total depth equal to $2 c$. The result for the comparison of systems 1 and 2 provides

$$
\widetilde{\Pi}_{21}^{2}=r_{2} / r_{1}=0.2
$$

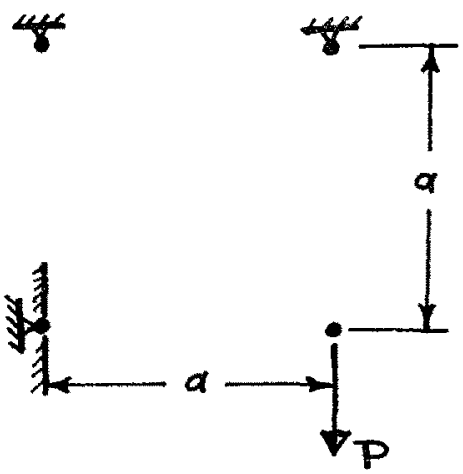

(a) Design Space
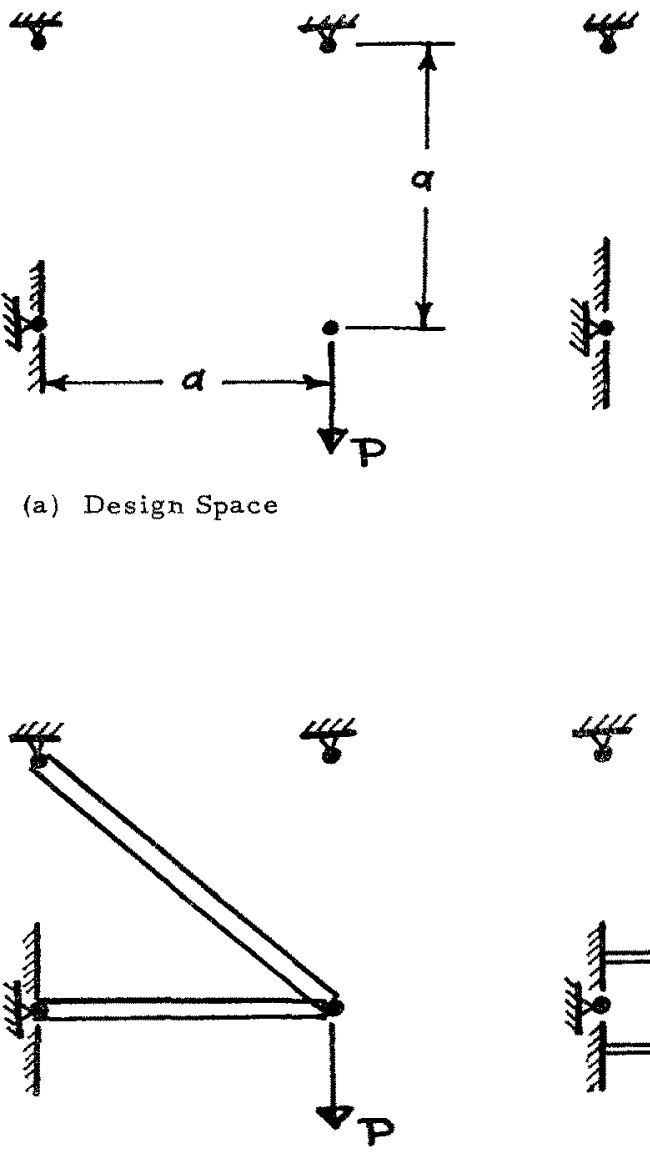

(c) System Two

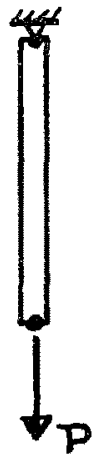

(b) System One

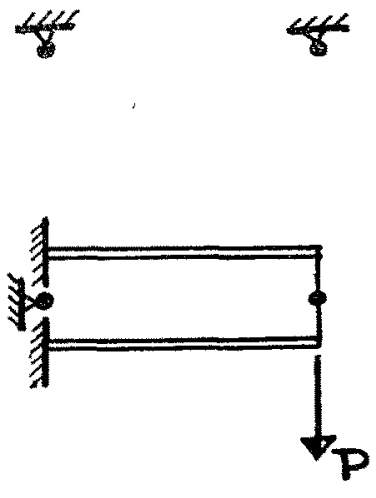

(d) System Three

(d) candidate systems. 
i.e., structure 2 will become economical only if its unit cost is less than one-fifth the unit cost of structure 1. The efficiency of the beam depends on its aspect ratio $c / a$. For the value $c / a=0.1$,

$$
\tilde{\Pi}_{31}^{2}=r_{3} / r_{1}=0.04,
$$

i.e., the beam is $1 / 25$ as efficient as direct transmission via system 1 .

\section{References}

1. Prager, W., Methods of Structural Optimization (to appear).

2. Sved, G., and Ginos, Z., Structural Optimization Under Multiple Loading, International Journal of Mechanical Sciences, Vol. 10, 1968.

3. Sheu, C. Y., and Scrmit, L. A., JR., Minimum Weight Design of Elastic Redundant Trusses Under Multiple Static Loading Conditions, AIAA Journal, Vol. 10, No. 2, 1972.

4. Nagtegal, J. C., and Prager, W., Optimal Layout of a Truss for Alternative Loads, International Journal of Mechanical Sciences, Vol. 15, 1973.

5. Prager, W., Optimality Criteria Derived from Classical Exiremum Principles, An Introduction to Structural Optimization, Edited by M. Z. Cohn, University of Waterloo, Waterloo, Ontario, Canada, 1968.

6. Drucker, D. C., and Shield, R. T., Bounds on Minimum Weight Design, Quarterly of Applied Mathematics and Physics, Vol. 15, No. 3, 1957.

7. Shreld, R. T., and PraGer, W., Optimal Structural Design for Given Deflection, Journal of Applied Mathematics and Physics, Vol. 21, 1970.

8. Masur, E. F., Optimum Stiffness and Strength of Elastic Structures, Proceedings of the ASCE, Journal of the Engineering Mechanics Division, Vol. 96-EM5, 1970.

9. Mroz, Z., Optimal Design of Structures of Composite Materials, International Journal of Solids and Structures, Vol. 6, No. 7, 1970.

10. Martin, J. B., The Optimal Design of Beams and Frames with Compliance Constraints, International Journal of Solids and Structures, Vol. 17, No. 3, 1971.

11. Taylor, J. E., Maximum Strength Elastic Structural Design, Proceedings of the ASCE, Journal of the Engineering Mechanics Division, Vol. 95-EM3, 1969. 